\title{
Cycloidal Computed Tomography
}

\author{
Charlotte K. Hagen $\odot,{ }^{*}$ Fabio A. Vittoria, ${ }^{\dagger}$ Oriol Roche i Morgó $\odot$, Marco Endrizzi, and \\ Alessandro Olivo $(0$ \\ Department of Medical Physics and Biomedical Engineering, University College London, Malet Place, \\ London WC1E 6BT, United Kingdom
}

(Received 31 July 2019; revised 16 June 2020; accepted 19 June 2020; published 23 July 2020; corrected 20 October 2020)

\begin{abstract}
A general concept is presented for increasing the in-slice spatial resolution in computed tomography in a flexible manner and with relatively large source focal spots and detector pixels. The flexibility is a result of probing the sample with an array of thin beamlets shaped by a mask. Provided that the mask apertures are smaller than the combined blur of the x-ray source and detector and that no significant overlap between beamlets occurs, this introduces higher spatial frequencies into the image-formation process. The application of a cycloidal acquisition scheme, by which the sample is simultaneously rotated and translated, facilitates an efficient exploitation of these frequencies and their reconstruction into high-resolution tomographic images. Additionally, a preliminary study of the signal-to-noise ratio versus the delivered dose reveals significant dose-saving potential.
\end{abstract}

DOI: 10.1103/PhysRevApplied.14.014069

\section{INTRODUCTION}

X-ray computed tomography [1-3] continues to have a transformative effect on clinical diagnostics, preclinical research, and nondestructive testing. A major strength of this imaging technique is the wide range of spatialresolution regimes that can be accessed (on the macro-, micro-, and even the nanoscale). While a high resolution is always desirable, its achievement requires dedicated hardware, which can be an inflexible constraint. The tomographic scanner must be equipped with an x-ray source with an adequately small focal spot and/or a detector with appropriately sized pixels that suffer little crosstalk [4]. This leaves few options for adaptive resolution adjustment besides exploiting geometrical magnification by changing the sample position along the beam path; here, a welldefined "sweet spot" is met when the projected source and pixel size are of comparable dimension when scaled to the sample isocenter [3]. While specialized $\mathrm{x}$-ray sources may feature a variable focal spot [5], their use is not the norm in a variety of applications. Likewise, detectors with small pixels, developed for high-resolution imaging, exist; however, these typically impose a small field of view, often

\footnotetext{
*charlotte.hagen.10@ucl.ac.uk

$\dagger$ Current address: ENEA-Radiation Protection Institute, 4 Via Martiri di Monte Sole, 40129 Bologna, Italy.

Published by the American Physical Society under the terms of the Creative Commons Attribution 4.0 International license. Further distribution of this work must maintain attribution to the author(s) and the published article's title, journal citation, and DOI.
}

no larger than a few centimeters or less. CCD cameras with variable optics can enable fast resolution switching but resolution is again coupled to the field of view; hence high-resolution imaging may only be possible locally for very small sections of the scanned sample. These aspects prove to be a restrictive factor, especially with an increasing number of applications requiring a flexible, ideally multiscale, approach to tomographic imaging.

Here, a mechanism is presented that enables increasing the in-slice resolution (at a constant slice thickness) in a flexible and efficient manner without having to utilize specialized sources and detectors and without relying on geometric magnification.

\section{METHODS}

The concept is enabled through the synergy of two advances in terms of the tomographic scanner layout and the data-acquisition strategy. Instead of by the full $x$ ray beam, the sample is probed by an array of beamlets generated by a mask with narrow slit-shaped apertures [Fig. 1(a)]. The mask period ( $p$ ), which defines the interbeamlet spacing, matches the effective (i.e., scaled to the sample isocenter) pixel size (a), i.e., $p=a / m$, where $m$ is a geometric scaling factor (for simplicity, the mask-sample distance is considered negligible). The mask must fulfill two design criteria; first, the aperture width $(w)$ must be smaller than the system blur, which is given by the convolution between the projected source distribution and the pixel response function. Second, $p$ must be sufficiently large so that any overlap of adjacent beamlets, where present, is only due to the tails of the source distribution. 


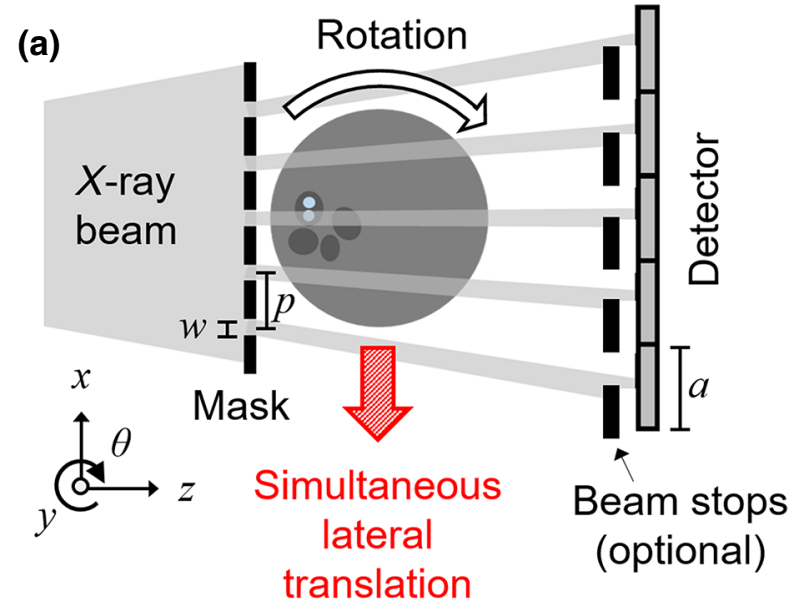

(b)

$$
\text { Sampling grid }
$$$$
\text { (rotation only) }
$$

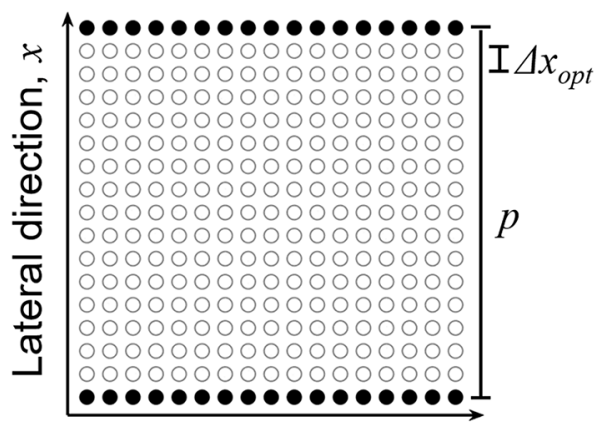

Angular direction, $\theta$ (c)
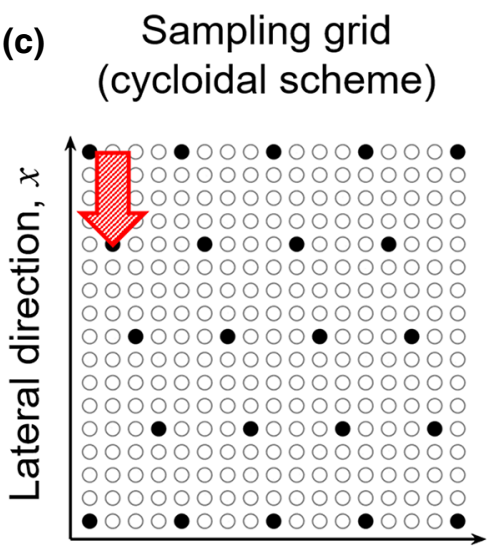

Angular direction, $\theta$
FIG. 1. (a) A schematic of cycloidal computed tomography (not to scale, seen from top); by adding an array of beam stops in front of the detector, the setup is transformed into an edge-illumination $\mathrm{x}$-ray phase-contrast imaging device. (b) A sinogram sampling grid for a rotation-only scheme. (c) A sinogram sampling grid for a cycloidal scheme. The grids are shown for one mask period and a subset of rotation angles; the combination of empty and filled circles shows the grids that would be achieved through fine lateral sampling (requiring dithering); the filled circles show the data that are sampled without dithering.
The latter does not impose a constraint on the pixel size, as one can divert from the $p=a / m$ relationship and employ a "line-skipping" mask where only every second (or third etc.) pixel column is illuminated. While the first criterion leads to the presence of additional spatial frequencies in the image-formation process (beyond the cutoff imposed by the source and the detector), the second criterion ensures that these frequencies are not encoded. To a first approximation, spatial frequencies up to the inverse of the aperture width $(1 / w)$ are now present, provided that diffraction effects and detector pixel crosstalk can be neglected [6]; note that the latter can always be made negligible through "line-skipping". Depending on the ratio $p / w$, these frequencies can be significantly higher than those that would be available without the subpixel beam fractioning.

In order to adequately exploit these extra spatial frequencies, the signal would normally need to be sampled with a step that is much finer than the mask period $\left(\Delta x_{\mathrm{opt}} \ll p\right)$ [7], which requires that the sample is scanned along the $x$ axis in several subpixel steps at each rotation angle, a frame is collected at each step, and all frames are combined into an upsampled projection. This procedure ("dithering") involves the acquisition of many more frames than if the sample were simply rotated. Since dead times are typically required for stop-starting the translation and rotation stages in between frames, this can lead to substantially increased scan times. However, if no dithering is applied, the large lateral sampling step $(\Delta x=p)$ prevents the available spatial frequencies from being accessed and reconstructed into high-resolution tomograms. We propose to combine a beamlet-based setup with an alternative acquisition scheme that does not involve dithering but that nevertheless allows us to exploit the available spatial frequencies. In this scheme, the sample is translated along the $x$ direction simultaneously with being rotated, corresponding to a "rototranslation" motion. This is a different procedure from dithering; while dithering involves a multistep scan, in a cycloidal acquisition the sample is shifted once per rotation angle (by a fraction of the total dithering distance). If the translation is unidirectional, each sample feature follows the trajectory of a cycloid. An equivalent, but perhaps more practical, implementation would be to translate the sample in one direction by up to one mask period, shift it back to its initial position, translate it again by up to one period, and so on. A cycloidal acquisition involves only a fraction of the frames of a dithered scan. Importantly, it can be implemented in a continuous (as opposed to step-and-shoot) 
fashion; as dead times are no longer required in this case, the scan time is determined by the exposure time only. To enable the reconstruction of tomographic images from cycloidally sampled data, a mathematical data-recovery technique is applied to fill the missing entries in the acquired sinogram. The cycloidal sampling is instrumental in this recovery step, as it is a means of breaking up the unbalanced arrangement of data points in the sinogram sampling grid that would result from a rotationonly scheme [Fig. 1(b)]. In the latter, the available data are densely packed along the angular-grid direction, but spaced far apart along the lateral direction, implying that any two neighboring projections provide little complementary information and therefore only marginally aid the data recovery. On the contrary, a cycloidal scheme leads to a more even spread of the available data [Fig. 1(c)], allowing us to adequately restore the missing data and gain access to spatial frequencies that would otherwise be lost.

As such, the presented concept belongs to a larger class of methods by which tomographic images are reconstructed from undersampled data. The focus of such methods has initially been on angular undersampling [8,9] but, more recently, and to some extent inspired by compressed sensing, it has shifted to lateral undersampling, e.g., the acquisition of incomplete projections by using "randomized" absorbing patterns in the beam path [10-12]. Cycloidal computed tomography differs from such approaches, as a highly structured illumination pattern is used and data recovery is not based on compressed sensing. While examples of a similarly structured beam fractioning exist, these focus to a large extent on dose reduction $[13,14]$ but not on increasing spatial resolution. Previous work aimed at high-resolution imaging $[15,16]$ involves relatively complex decoding or deconvolution-type algorithms, which are not required by our approach. An additional difference is that cycloidal computed tomography offers a simple solution for switching from attenuation into phase-contrast mode, allowing us to increase feature detectability when necessary, e.g., for samples that are composed of similar or low-atomic-number materials, such as soft biological tissue [17]. For example, by adding an array of beam stops in front of the detector [Fig. 1(a)], the setup is transformed into an edgeillumination x-ray phase-contrast imaging device [18]. In such a system, the beamlets are initially aligned with the edges of the beam stops, causing a certain (reference) intensity to fall onto the pixels. When a sample is inserted, refraction causes minute changes to the beamlets' directions, which either increases or decreases the detected intensity per pixel. This sensing mechanism is incoherent (in the physical wave-optics sense) [19] and achromatic [20] and therefore compatible with standard xray tubes with relatively large focal spots and broad energy spectra.

\section{RESULTS}

Cycloidal computed tomography is demonstrated using a setup comprising a MicroMax-007 HF x-ray tube (Rigaku, Japan) with a rotating molybdenum anode, operated at $40 \mathrm{kVp}$ and $25 \mathrm{~mA}$, resulting in a horizontal focal spot of approximately $70 \mu \mathrm{m}$ full wave at half maximum (FWHM). The detector is the Pixirad-2 photon counter, with a pixel size of $62 \mu \mathrm{m}$. The mask, fabricated by electroplating gold strips onto a graphite substrate (Creatv Microtec, USA), has a $79-\mu \mathrm{m}$ period and $10-\mu \mathrm{m}$ apertures. The source-to-mask and mask-to-detector distances are $1.6 \mathrm{~m}$ and $0.93 \mathrm{~m}$, respectively. With these distances, the mask period covers two pixel columns when projected to the detector, which is equivalent to employing a detector with pixels that are twice as large $(124 \mu \mathrm{m}$ in this case). This setup is designed to fulfill the working criteria of the method: (i) the mask apertures $(w)$ are smaller than both the effective projected source focal spot $(26 \mu \mathrm{m}$ FWHM) and the effective pixel size $(80 \mu \mathrm{m})$; (ii) the width of the beamlets, when magnified to the detector plane and broadened by the extended source, is approximately $44 \mu \mathrm{m}$ (FWHM). As this is much smaller than the magnified $p$, an effective separation between beamlets is achieved. Data are acquired for a custom-built phantom (polyethylene spheres, diameter 425-500 $\mu \mathrm{m}$ ) and a biological specimen (rabbit esophagus) placed in plastic straws (3-mm and 5.5-mm diameter, respectively). A detailed description of the biological sample can be found elsewhere [21]. The sample stage is positioned at approximately $2 \mathrm{~cm}$ downstream of the mask, so that the mask period is $80 \mu \mathrm{m}$ when scaled to the sample isocenter. Scans are performed in phase-contrast mode, which entails placing an array of beam stops (in practice, another mask with a $98-\mu \mathrm{m}$ period and $17-\mu \mathrm{m}$ apertures) at approximately $2 \mathrm{~m}$ from the source. The two masks have a $10-\mu \mathrm{m}$ lateral offset from each other.

For a proof of concept, dithered data sets are acquired and subsequently subsampled to mimic rotation-only and cycloidal acquisitions. The dithered scans involve eight lateral scanning steps (10 $\mu \mathrm{m}$ each) per rotation angle; since $w \approx p / 8$, this is the number of steps required to ensure that samples are entirely illuminated. Data are collected in $0.2^{\circ}$ angular steps over $180^{\circ}$, corresponding to 900 projections $(900 \times 8=7200$ frames $)$ in total. The exposure time per frame is $2 \mathrm{~s}$. To obtain rotation-only data, all but the frames acquired during the first dithering steps are discarded. To generate cycloidal data, again all but the frames from a single dithering step are discarded, but now this is a different step for each angle, corresponding to a different lateral sample translation [specifically, the first dithering step is kept at the first angle, the third step at the second angle, the fifth step at the third angle, the seventh step at the fourth angle, and so on; this sequence is repeated to generate an interlaced pattern like the one 

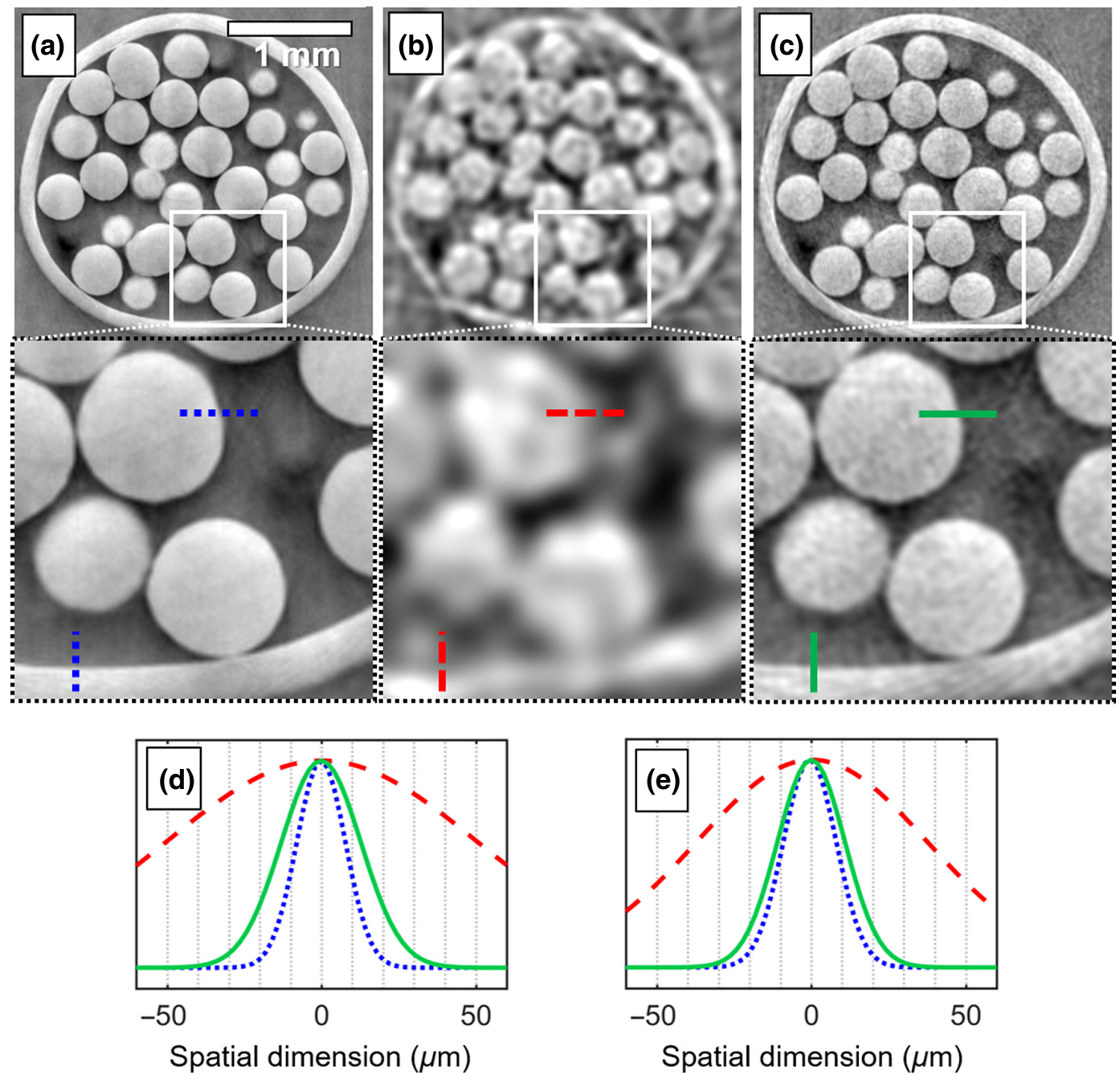

FIG. 2. Tomographic images of (425-500)- $\mu$ m-diameter polyethylene spheres, acquired with (a) dithering, (b) rotation-only, and (c) cycloidal ("rototranslation") sampling (see text for description). (d),(e) Line-spread functions (LSFs) extracted across (d) horizontal and (e) vertical edges in the dithered (dotted line), rotation-only (dashed), and cycloidal (solid line) images.

shown in Fig. 1(c)]. The rotation-only and cycloidal data both comprised 900 frames. Following dark- and flatfield corrections, bivariate cubic interpolation is applied to both sinograms to recover the missing entries. This is followed by phase retrieval [22], which converts the refraction-based edge signal into area contrast, and tomographic reconstruction using filtered back projection. The dithered images are processed in the same way but without the interpolation step.

The results are shown in Figs. 2 (spheres) and 3 (rabbit esophagus). Notably, cycloidal sampling leads to a resolution almost as high as dithering, despite being reconstructed from an eighth of the frames. In comparison, the rotation-only images are significantly blurred. Line spread functions (LSFs), obtained by fitting and differentiating error functions to horizontal and vertical edges in the sphere sample, confirm these observations. The in-slice resolution (estimated from the FWHM of the respective LSF) in the cycloidally sampled image (30 $\mu \mathrm{m}$ horizontally, $26 \mu \mathrm{m}$ vertically) is only slightly below that in the dithered image (19 $\mu \mathrm{m}$ horizontally, $20 \mu \mathrm{m}$ vertically). In comparison, the in-slice resolution in the rotation only image is severely degraded (119 $\mu$ m horizontally, 88 $\mu \mathrm{m}$ vertically). It should also be noted that these results demonstrate the ability of the beamlet approach to provide access to resolutions beyond the effective projected source and pixel size; notably, a resolution of $20 \mu \mathrm{m}$ is achieved with (effectively) $124 \mu \mathrm{m}$ pixels while only marginally relying on magnification (the magnification between sample and detector is 1.55). Figure 3 allows for a more qualitative evaluation of the concept. The sample originates from research into organ decellurization for tissue-engineering applications and while detailed information on this topic can be found elsewhere [23], it should 

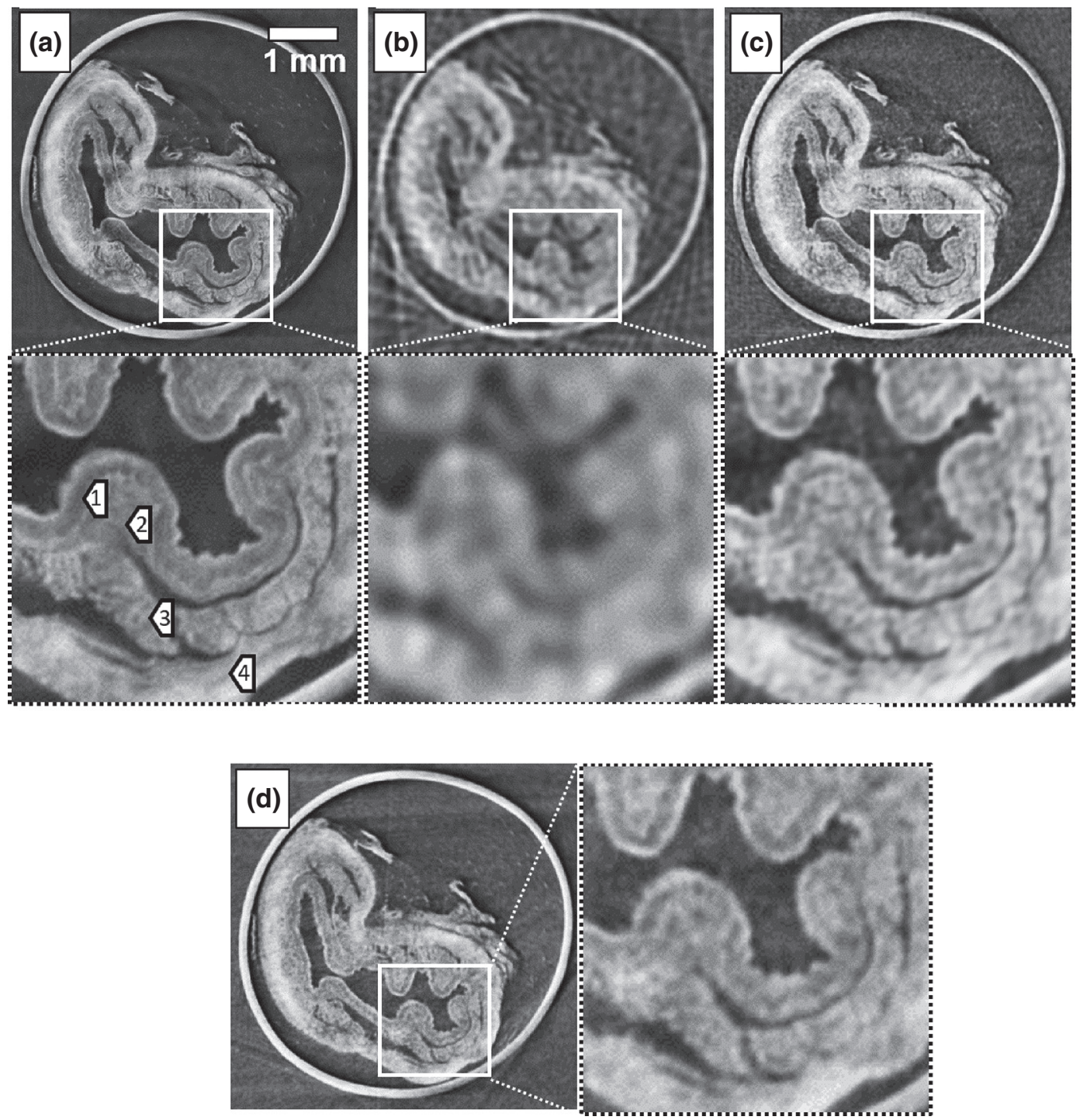

FIG. 3. Tomographic images of a rabbit esophagus, acquired with (a) dithering, (b) rotation-only sampling, (c) cycloidal ("rototranslation") sampling, and (d) cycloidal ("rototranslation") sampling in continuous mode. The arrows in the dithered image refer as follows: 1, mucosa; 2, submucosa; 3, muscularis propria; 4, adventitia.

be noted that the ability to preserve a tissue's microstructural integrity during this process is considered an indicator for good performance. The dithered image provides the necessary spatial resolution for such an assessment, as major anatomical structures of the esophagus can be seen and distinguished. This information is almost entirely lost with a rotation-only scheme. In contrast, when applying cycloidal sampling, resolution is recovered to a level that permits an effective visualization of the anatomy.

Figure 3(d) shows a cycloidally sampled image acquired in continuous mode (i.e., the rotation and translation are performed without interruption). During this scan, the sample is translated at a speed of $10 \mu \mathrm{m} / \mathrm{s}$, while simultaneously being rotated at a speed of $0.1^{\circ}$ per second (over a total range of $180^{\circ}$ ). The exposure time per frame is again
$2 \mathrm{~s}$, resulting in an angular increment of $0.2^{\circ}$ (900 frames). The data are processed as above, besides the fact that the sinogram has to be regridded (i.e., data points are "shifted back" by the respective sample translation distance per angle) before the interpolation to restore the correct sample geometry. Since dead times are negligible in this case, the scan time is identical to the total exposure time (30 min). No notable degradation of image quality is observed relative to the step-and-shoot data shown in Fig. 3(c).

\section{CONCLUSION}

In summary, cycloidal computed tomography offers several advantages. First, it allows the in-slice resolution to be increased largely independently of the main scanner 
hardware, as it effectively decouples this metric from the $\mathrm{x}$-ray source and detector characteristics; rather, it is only required that a carefully designed mask is included in the setup. This provides flexibility and enables fast resolution switching, e.g., by including masks with different apertures on the same frame or by using several masks with increasingly narrow apertures in succession. Both provide access to increasingly fine resolution levels, hence opening new opportunities for multiscale tomographic imaging. Second, a greater independence between the spatial resolution and the field of view is achieved; masks with apertures as narrow as a few micrometers can readily be manufactured on a $10 \times 10 \mathrm{~cm}^{2}$ wafer by x-ray or even UV lithography, $20 \times 20 \mathrm{~cm}^{2}$ masks can be made by "stitching" individual wafers, and larger mask dimensions could be achieved by alternative techniques such as laser ablation. Third, the proposed "rototranslation" scheme is a means for efficient high-resolution scanning, as scans can be performed continuously, which eliminates dead times.

In addition, the fact that large portions of the sample are shielded from radiation during scans offers opportunities for dose reduction. Evidence for the dose-saving capabilities of cycloidal computed tomography are provided in the Appendix, where we analyze how the signal-to-noise ratio (SNR) in cycloidal images compares to that in a dithered image when the dose in the former is gradually increased to the level of the latter. The results, although preliminary, show great potential: the SNR in cycloidal images is much higher than in their dithered counterpart, even when the dose is lower. Cycloidal computed tomography may therefore become an attractive scanning technique for biomedical applications, where high-resolution images are needed while it is crucial that the dose is kept at a minimum. This may have a positive impact on the future role of x-ray computed tomography in such disciplines, e.g., it could enable more extensive longitudinal preclinical studies.

From a practical point of view, different options for implementing cycloidal acquisitions are available. The most straightforward one is to collect data in a step-andshoot fashion and translate the sample by up to one mask period, then shift it back, translate it again, and so on. Such a sequence has the advantage that data processing is relatively simple, as the correct sample geometry is maintained. However, a faster method would be to translate and rotate the sample continuously. A unidirectional translation would require a mask and detector large enough to cover the sample both when it is in its initial position and after it has completed its full lateral translation. A more general solution may be to "continuously" translate the sample back and forth. This would imply a nonconstant speed of the sample translation stage, as repeated changes of the translation direction would require periods of motor acceleration and deceleration, which could, however, be accounted for as part of the data processing.
Finally, the presented concept is expandable so as to also flexibly and efficiently increase the spatial resolution outside the in-slice plane. While the results presented here are obtained using a mask with slit-shaped apertures and horizontal "rototranslation" sampling, masks with square or circular apertures, creating pencil beams rather than bladelike beamlets, would also make the resolution-enhancing capabilities of such a setup available along the vertical direction. Appropriate sampling trajectories would need to be developed and combined with adequate mathematical data-recovery methods. Alternatively, sampling and reconstruction schemes that can provide isotropic resolution images from measurements with unisotropic x-ray sources [24] may also be applicable to recover vertical resolution in the case of bladelike beamlets.

\section{ACKNOWLEDGMENTS}

C.K.H. and M.E. are supported by the Royal Academy of Engineering (RAEng) under the Research Fellowship scheme; A.O. is supported by the RAEng under the Chair in Emerging Technologies scheme. Additional support from UK Research and Innovation (UKRI) (Grant No. EP/T005408/1) is acknowledged. We would like to thank Panagiotis Maghsoudlou and Paolo De Coppi for the kind preparation and provision of the esophagus specimen used in this study.

\section{APPENDIX: DOSE-REDUCTION POTENTIAL}

We present additional experimental and simulated results as early evidence of the dose-saving capabilities of the cycloidal computed tomography concept. The additional images are compared in terms of their SNR and thus complement the images shown in Fig. 2 in the main text, which are compared in terms of their spatial resolution. Specifically, we analyze how the SNR in cycloidally sampled images compares to the SNR in a dithered image when the dose in the former is gradually increased to the level of the latter.

\section{Experimental results}

The additional experimental data are acquired with a setup featuring a MicroMax-007 HF x-ray tube (Rigaku, Japan) with a rotating molybdenum target operated at $40 \mathrm{kVp}$ and $10 \mathrm{~mA}$. The $\mathrm{x}$-ray beam is structured into an array of beamlets via a mask with a $79-\mu \mathrm{m}$ period and 12 $\mu \mathrm{m}$ apertures. The mask is positioned at approximately 0.7 $\mathrm{m}$ downstream of the source. The detector is a CMOSbased flat-panel C9732DK-11 (Hamamatsu, Japan) with directly deposited CsI and a 50- $\mu \mathrm{m}$ pixel size. The sourceto-detector distance is $0.875 \mathrm{~m}$. With these distances, the mask period spans over two pixel columns when projected to the detector. Data are acquired for the same custom-built 
phantom as in the main text, which consists of polyethylene spheres with a (425-500)- $\mu$ m diameter contained in a plastic straw (3-mm diameter). The sample stage is located at $2 \mathrm{~cm}$ downstream of the mask.

A multiframe dithered data set of the phantom is acquired that is subsequently subsampled to mimic cycloidal acquisitions. The scan involves scanning the sample laterally in eight steps (10 $\mu \mathrm{m}$ each) per rotation angle and collecting eight frames per step (to enable the creation of images corresponding to different delivered doses by including an increasing number of frames when reconstructing cycloidal images). This is repeated for 900 angular projections, for which the sample is rotated in $0.2^{\circ}$ steps over a range of $180^{\circ}$. In total, $900 \times 8 \times 8=57600$ frames are acquired. The exposure time per frame is $1.1 \mathrm{~s}$.

First, a dithered image of the phantom is reconstructed by considering all dithering steps but including only one frame per step. The reconstruction involves dark- and flat-field corrections and tomographic reconstruction using filtered back projection. The dithered image, obtained from $900 \times 8=7200$ frames, is shown in Fig. 4(a). Next, to generate cycloidal data, only a single dithering step is included but this is a different step (corresponding to a different lateral sample offset) at each rotation angle (the same subsampling procedure as described in the main text is applied). Eight cycloidal images are then reconstructed by including an increasing number of frames (ranging from one to eight frames) per angle, which are averaged. Besides dark- and flat-field corrections, averaging of frames, and tomographic reconstruction using filtered back projection, bicubic interpolation is applied to the cycloidal sinograms so as to restore the missing entries caused by the interlaced sampling pattern. The cycloidal images are shown in Figs. 4(c)-4(j). These images are reconstructed from 900, 1800, 2700, 3600, 4500, 5400, 6300 , and 7200 frames, respectively, which corresponds to $12.5 \%, 25 \%, 37.5 \%, 50 \%, 62.5 \%, 75 \%, 87.5 \%$, and $100 \%$ of the dose of the dithered image shown in Fig. 4(a). For comparison, we also reconstruct a rotation-only image, by including, for each angle, only the first dithering step in the reconstruction. All frames at this dithering step are considered, such that the rotation-only image is reconstructed from 7200 frames, i.e., $100 \%$ of the dose of the dithered image. The rotation-only image is reconstructed in the same way as the cycloidal images, i.e., by applying darkand flat-field corrections, bicubic interpolation, and filtered back projection. The rotation-only image is shown in Fig. 4(b).

Contrary to the data in the main text, the images shown here are acquired in attenuation-only instead of phasecontrast mode (i.e., no beam-stop array is used). As polyethylene spheres exhibit weak x-ray attenuation, the images are therefore much noisier than those in Fig. 2. The reason for not acquiring the images in phase-contrast mode is that this Appendix is dedicated to demonstrating the
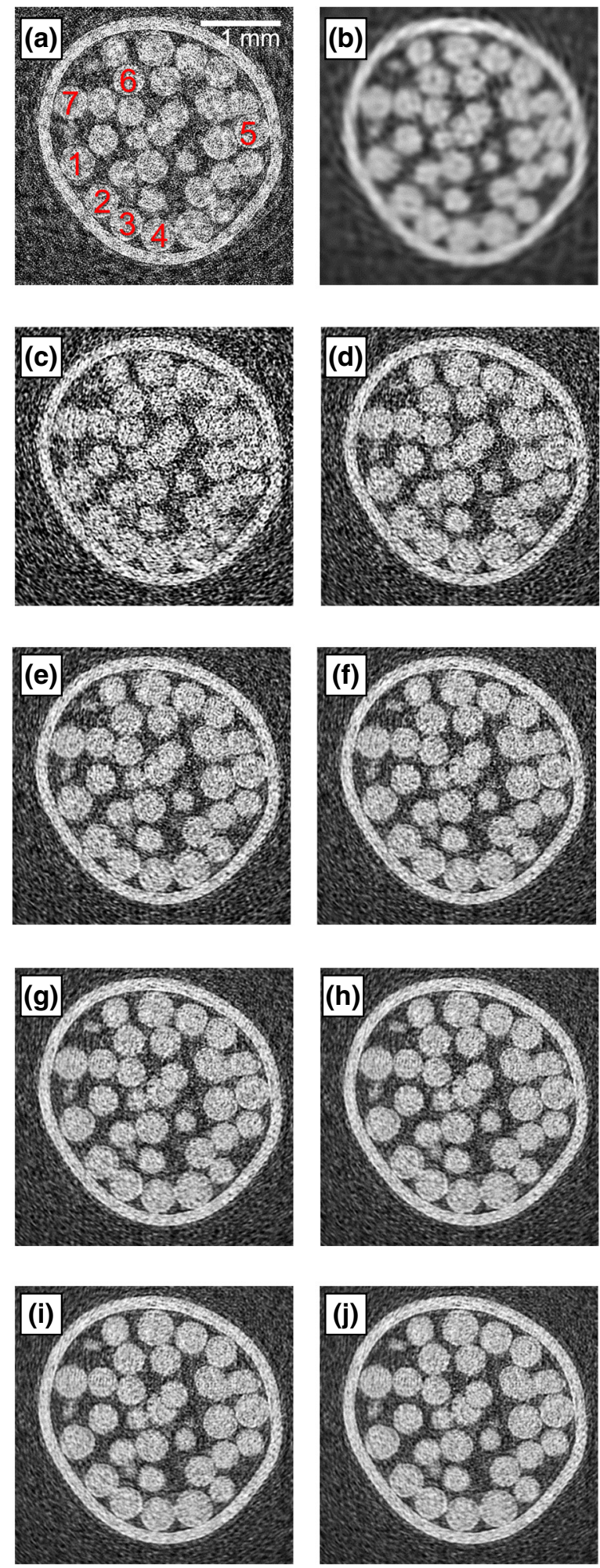

FIG. 4. Tomographic images of (425-500)- $\mu$ m-diameter polyethylene spheres, acquired with (a) dithering, (b) rotationonly sampling, and (c-j) cycloidal ("rototranslation") sampling. The images in panels (c)-(j) are reconstructed from an increasing number of frames, corresponding to an increasing fraction of the dose delivered in the dithered image: (c) $12.5 \%$ dose; (d) $25 \%$ dose; (e) $37.5 \%$ dose; (f) $50 \%$ dose; (g) $62.5 \%$ dose; (h) $75 \%$ dose; (i) $87.5 \%$ dose; (j) $100 \%$ dose. 


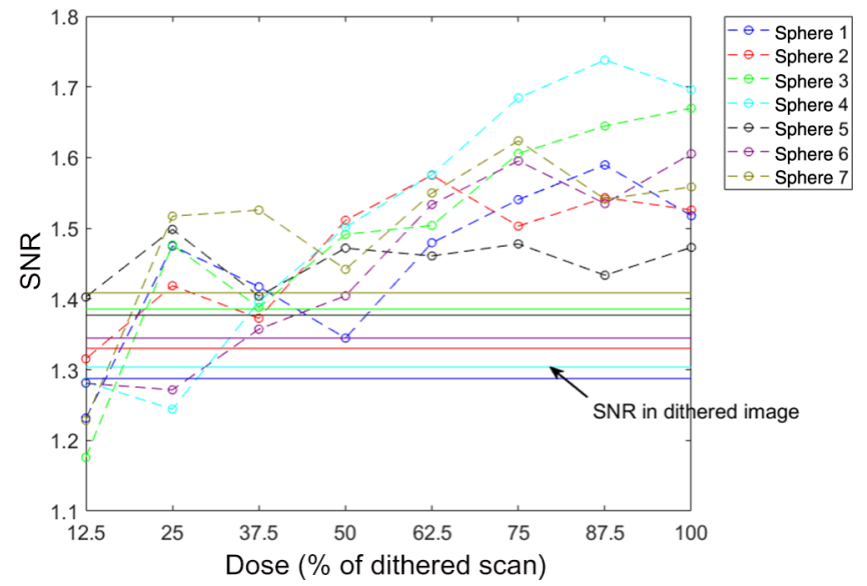

FIG. 5. The SNR for seven polyethylene spheres [see Fig. 4(a)] obtained when applying cycloidal sampling (dashed lines). The values are plotted as a function of their dose, expressed as a percentage of the dose of the dithered image. The corresponding SNR values for the respective spheres in the dithered image are shown as horizontal lines (solid lines).

dose-noise performance of the cycloidal computed tomography concept, which, at its core, is enabled through combining a carefully structured $\mathrm{x}$-ray beam with a cycloidal sampling scheme; access to phase contrast is an optional additional advantage. Scanning in phase-contrast mode requires applying a phase-retrieval procedure [22] during image reconstruction, which consists of applying a lowpass filter to the images and therefore effectively eliminates noise above a certain spatial frequency; hence, the phaseretrieval step affects the noise performance of the method. The attenuation-only mode is chosen to exclude this effect from the analysis and to facilitate a straightforward interpretation of the data.

To analyze the SNR values in the eight cycloidal images shown in Figs. 4(c)-4(j) and compare them, as a function of their dose, to the SNR in the dithered image in Fig. 4(a), regions of interest (containing between 180 and 306 pixels, depending on the size of the sphere) are extracted for eight spheres in the phantom [see Fig. 4(a)], and their means and standard deviation are calculated. The obtained values for the SNR, defined as the mean divided by the standard deviation, are plotted in Fig. 5. The values extracted from the cycloidal images are shown as dashed lines, while the corresponding values obtained from the dithered image are shown as solid lines. It can be seen that in the cycloidal images, on average, the SNR increases as a function of the dose. This is not surprising, as noise is reduced when more frames are averaged. It can also be seen that the SNR curves obtained from the cycloidal images are mostly above those in the dithered image, which shows that, at the same dose, cycloidal sampling leads to a higher SNR than dithering. Alternatively, cycloidal sampling allows
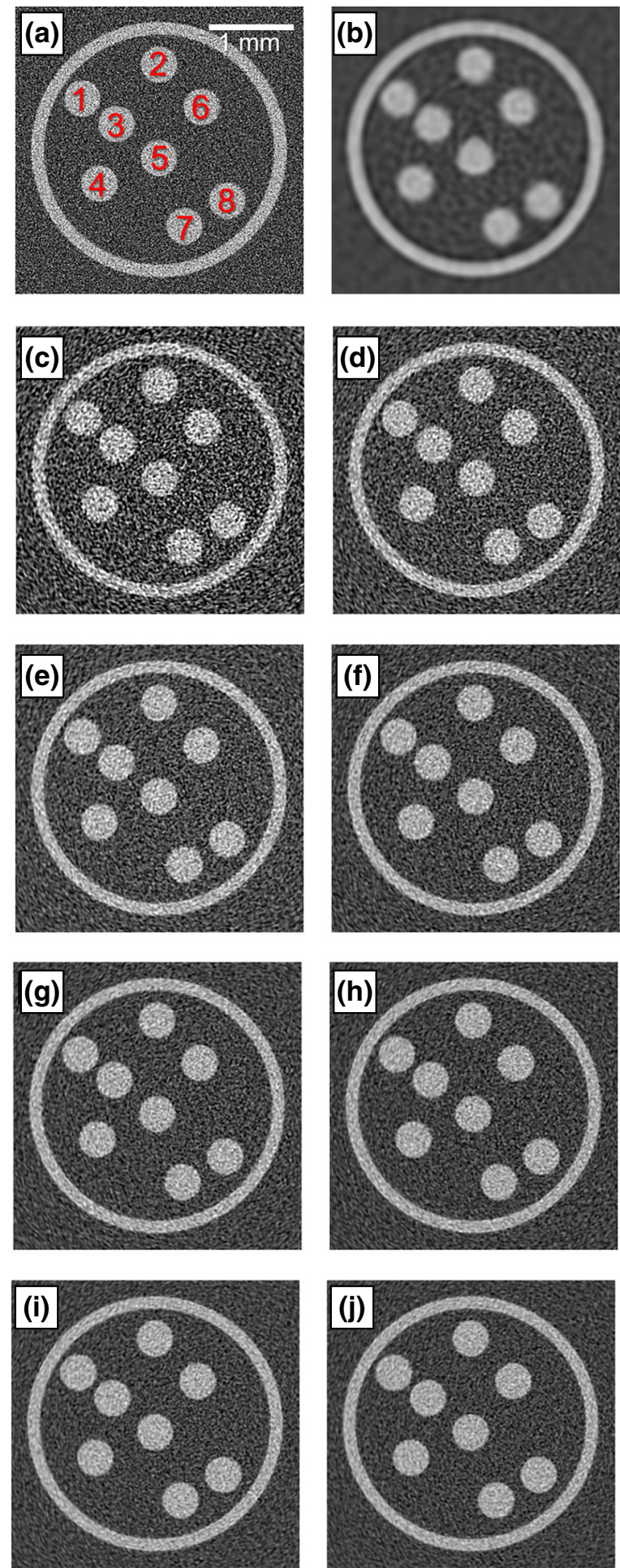

FIG. 6. Simulated tomographic images of the numerical phantom, acquired with (a) dithering, (b) rotationonly sampling, and (c)-(j) cycloidal ("rototranslation") sampling. The images in panels (c)-(j) are simulated assuming an increasing number of photons, corresponding to an increasing fraction of the dose delivered in the dithered image: (c) $12.5 \%$ dose; (d) $25 \%$ dose; (e) $37.5 \%$ dose; (f) $50 \%$ dose; (g) $62.5 \%$ dose; (h) $75 \%$ dose; (i) $87.5 \%$ dose; (j) $100 \%$ dose. 


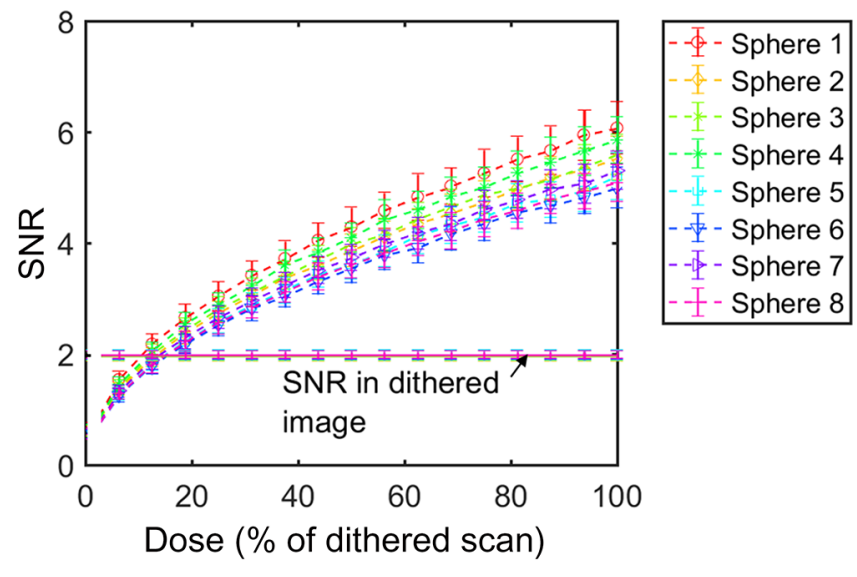

FIG. 7. Simulated SNR results for the eight spheres in the numerical phantom, obtained when applying cycloidal sampling (dashed lines). The values are plotted as a function of the dose, expressed as a percentage of the dose of the dithered image. The corresponding SNR values for the respective spheres in the dithered image are shown as horizontal lines (solid lines).

the same SNR to be achieved at a lower dose, which shows the dose-saving capabilities of the method.

\section{Simulated data}

We further investigate the dose-saving capabilities of cycloidal computed tomography using an experimentally validated simulation of the beamlet-based imaging approach [25]. The setup and acquisition parameters used in the simulation are chosen so as to match the experiment for which data are reported above (as the only differences, a monochromatic beam of $18 \mathrm{keV}$ and a "perfect" photoncounting detector are assumed). Data are simulated for a numerical phantom consisting of eight $425-\mu \mathrm{m}$-diameter spheres in a cylinder, so as to resemble the experimental phantom. First, a dithered scan is simulated assuming $N=10^{4}$ photons per beamlet and applying Poisson noise to the data. Next, cycloidal scans, consisting of eight times fewer data points than the dithered scans, are simulated; the simulation is repeated several times while the number of photons per beamlet is gradually increased until the dose matches $100 \%$ of the dose applied in the dithered scan. For comparison, a rotation-only acquisition at $100 \%$ of the dithered scan is also simulated. All simulations are repeated 100 times to obtain meaningful statistics. Dithered, cycloidal, and rotation-only images are reconstructed in the same way as described above. The results are shown in Fig. 6.

Analogously to the experimental data, the SNR in the cycloidal images shown in Figs. 6(c)-4(j) is extracted for each sphere and plotted as a function of the dose (Fig. 7). Again, the corresponding SNR values in the respective spheres in the dithered image are plotted as horizontal lines. The data points show the mean of all SNR values obtained from the 100 repetitions of each simulation and the error bars show the standard deviation thereof. As in the plot resulting from the experiment, it can be seen that SNR increases with the dose for all spheres. In fact, the curves appear to follow a $\sqrt{N}$ trend, which is expected in the presence of Poisson noise (note that the curves obtained from the experimental data do not strictly follow this trend because we use an indirect detector, which leads to a non-Poissonian dark-current contribution to the noise). Again, the fact that the SNR curves obtained from the cycloidal images are mostly above those in the dithered image implies that, at the same dose, a higher SNR can be achieved with cycloidal sampling or, alternatively, the same SNR can be achieved at a lower dose.

\section{Conclusion}

In summary, the additional data and analysis presented in this Appendix support the conclusion that cycloidal computed tomography enables dose reductions simultaneously with being a means for flexibly increasing spatial resolution [this is again shown through the comparison of rotation-only and cycloidal images in Figs. 4(b), 6(b) and Figs. 4(j), 6(j)] and reducing scan times. At the same time, we would like to emphasize that SNR is a somewhat limited metric when discussing image quality, as it does not provide information on the noise-power spectrum. One may infer from the images shown in Figs. 4(c)-4(j) and Figs. 6(c)-6(j) that the interpolation step, which is part of the reconstruction of cycloidal images, alters the noise-power spectrum. Specifically, the noise-power spectrum appears to be weighted toward lower spatial frequencies, as the cycloidal images have a slightly "blobby" noise texture. We anticipate that further studies using metrics such as the root mean square error (RSME) or the structured similarity index (SSIM) will lead to a refined evaluation of the performance of the cycloidal computed tomography approach.

[1] S. Webb, The Physics of Medical Imaging (CRC Press, Boca Raton, 1988).

[2] A. Kak and M. Slaney, Principles of Computerised Tomographic Imaging (IEEE, New York, 1988).

[3] T. Buzug, Computed Tomography; From Photon Statistics to Modern Cone-Beam CT (Springer, Berlin, Heidelberg, 2008).

[4] J. Wang and D. Fleischmann, Improving spatial resolution at CT: Development, benefits, and pitfalls, Radiology 289, 262 (2018).

[5] J. Grimes, X. Duan, L. Yu, A. Halaweish, N. Haag, S. Leng, and C. McCollough, The influence of focal spot 
blooming on high-contrast spatial resolution in CT imaging, Med. Phys. 42, 6011 (2015).

[6] P. Diemoz, F. Vittoria, and A. Olivo, Spatial resolution of edge illumination x-ray phase contrast imaging, Opt. Express 22, 15514 (2014).

[7] C. K. Hagen, F. A. Vittoria, M. Endrizzi, and A. Olivo, Theoretical Framework for Spatial Resolution in EdgeIllumination X-Ray Tomography, Phys. Rev. Appl. 10, 054050 (2018).

[8] R. Rangayyan, A. P. Dhawan, and R. Gordon, Algorithms for limited-view computed tomography: An annotated bibliography and a challenge, Appl. Optics 24, 4000 (1985).

[9] X. Yang, R. Hofmann, R. Dapp, T. van de Kamp, T. dos Santos Rolo, X. Xiao, J. Moosmann, J. Kashef, and R. Stotzka, TV-based conjugate gradient method and discrete L-curve for few-view CT reconstruction of x-ray in vivo data, Opt. Express 23, 5368 (2015).

[10] A. Olivo and R. Speller, Coded aperture computed tomography, Proc. SPIE 7468, 74680B (2009).

[11] Y. Kaganovsky, D. Li, A. Holmgren, H. Jeon, K. P. MacCabe, D. G. Politte, J. A. O'Sullivan, L. Carin, and D. J. Brady, Compressed sampling strategies for tomography, J. Opt. Soc. Am. A 31, 1369 (2014).

[12] D. J. Brady, A. Mrozack, K. MacKabe, and P. Llull, Compressive tomography, Adv. Opt. Photonics 7, 756 (2015).

[13] S. Cho, T. Lee, J. Min, and H. Chung, Feasibility study on many-view under-sampling technique for low-dose computed tomography, Opt. Eng. 51, 090501 (2012).

[14] A. P. Cuadros and G. R. Arce, Coded aperture optimization in compressive X-ray tomography: A gradient descent approach, Opt. Express 25, 23833 (2017).

[15] Y. Zhu, D. Chen, Y. Zhao, H. Li, and P. Zhang, An approach to increase the resolution of industrial CT images based on an aperture collimator, Opt. Express 21, 27946 (2013).

[16] E. Mojica, S. Pertuz, and H. Arguello, High-resolution coded-aperture design for compressive x-ray tomography using low-resolution detectors, Opt. Commun. 404, 103 (2017).

[17] A. Momose, Development toward high-resolution x-ray phase imaging, Microscopy 66, 155 (2017).
[18] A. Zamir, C. K. Hagen, P. C. Diemoz, M. Endrizzi, F. A. Vittoria, Y. Chen, M. A. Anastasio, and A. Olivo, Recent advances in edge illumination X-ray phase-contrast tomography, J. Med. Imaging 4, 040901 (2017).

[19] A. Olivo and R. Speller, A coded-aperture technique allowing $\mathrm{x}$-ray phase contrast imaging with conventional sources, Appl. Phys. Lett. 91, 074106 (2007).

[20] M. Endrizzi, F. A. Vittoria, G. Kallon, D. Basta, P. C. Diemoz, A. Vincenzi, P. Delogu, R. Bellazzini, and Alessandro Olivo, Achromatic approach to phase-based multi-modal imaging with conventional x-ray sources, Opt. Express 23, 16473 (2015).

[21] C. K. Hagen, P. Maghsoudlou, G. Totonelli, P. C. Diemoz, M. Endrizzi, L. Rigon, R. H. Menk, F. Arfelli, D. Dreossi, E. Brun, P. Coan, A. Bravin, P. De Coppi, and A. Olivo, High contrast microstructural visualization of natural acellular matrices by means of phase-based x-ray tomography, Sci. Rep. 5, 18156 (2015).

[22] P. C. Diemoz, C. K. Hagen, M. Endrizzi, M. Minuti, R. Bellazzini, L. Urbani, P. De Coppi, and A. Olivo, Single-Shot $\mathrm{X}$-Ray Phase-Contrast Computed Tomography with NonMicrofocal Laboratory Sources, Phys. Rev. Appl. 7, 044029 (2017).

[23] G. Totonelli, P. Maghsoudlou, J. M. Fishman, G. Orlando, T. Ansari, P. Sibbons, M. A. Birchall, A. Pierro, S. Eaton, and P. De Coppi, Esophageal tissue engineering: A new approach for esophageal replacement, World J. Gastrointero. 18, 6900 (2012).

[24] M. Vassholz, B. Koberstein-Schwarz, A. Ruhlandt, M. Krenkel, and T. Salditt, New X-Ray Tomography Method Based on the 3D Radon Transform Compatible with Anisotropic Sources, Phys. Rev. Lett. 116, 088101 (2016).

[25] F. A. Vittoria, P. C. Diemoz. M. Endrizzi, L. Rigon, F. C. Lopez, D. Dreossi, P. R. T. Munro, and A. Olivo, Strategies for efficient and fast wave optics simulation of codedaperture and other $\mathrm{x}$-ray phase-contrast imaging methods, Appl. Optics 52, 6940 (2013).

Correction: A Grant Number in the Acknowledgment section contained an error and has been fixed. 